\title{
Application of automated liquid handling in ligand-binding assay-based bioanalytical method development: the practical considerations
}

\author{
Mark Ware ${ }^{1}$, Gopi Shankar ${ }^{1}$, Patrick Breslin ${ }^{1}$ \& Tong-Yuan Yang*,1 \\ ${ }^{1}$ Biologics Development Sciences, Janssen BioTherapeutics, Janssen R\&D LLC., Spring House, PA 19477, USA \\ * Author for correspondence: TYang9@its.jnj.com
}

First draft submitted: 14 September 2017; Accepted for publication: 29 January 2018; Published online: 21 March 2018

Keywords: automated liquid handling • automation • bioanalysis $\bullet$ bioanalytical $\bullet$ ligand-binding assay

The ligand-binding assay (LBA) is the standard bioanalytical methodology to support preclinical and clinical development of biologics. LBA methods are based on molecular interactions of specific and selective critical reagents, which are large proteins themselves, with intended analyte in often a complex biological matrix such as serum. There are several benefits of automation in LBA method development. Primarily the replacement of manual with automated liquid handling will result in improved accuracy and precision of the assay [1], reduced human errors and lowering of the physical stress due to manual steps conducted by bench scientists. The decreased variation in pipetting that will then be achieved leads to the generation of higher quality bioanalytical data having greater reproducibility within studies and therefore more comparability across studies over time. This commentary will highlight our experience in applying automated liquid handling to each of these critical components of LBA method development process in bioanalytical laboratories conducting pharmacokinetic (PK), anti-drug antibody (ADA) and target engagement (TE) method development using LBAs, from start of critical reagent screening/pairing, to reagent addition, to sample/quality control (QC) dilutions, preparation of standard calibrators and QC samples with a focus on implementation in regulated bioanalysis.

\section{Selection of automation platforms}

There is a broad variety of automated liquid handling systems available in the market today. They mainly are either air-based system or liquid-filled system for pipetting with either/or a combination of fixed and disposable tips. Automation platforms with liquid detection system are used to not only provide a record of action of aspiration but also to aid when the volumes of bioanalytical samples are low.

Numerous publications have promoted using automated robotic systems in bioanalytical laboratories with different platforms and logistics of set-up [2-7]. The 'total laboratory automation (TLA)' proposed for routine clinical chemistry laboratories [4] is not always the best strategy for the application of automation to the development of PK, ADA and TE methods based on LBA methods. Most of the leading manufacturers of integrated robotic and automated liquid handling systems have produced LBA-based systems and have the flexibility to work with multiple assay platforms, run multiple methods in a single operation and are designed to take advantage of the vertical space within a laboratory to achieve the capacity of functionality required.

In our laboratory, we took the initial path of purchasing modular automated liquid handling systems which can serve several benefits to our bioanalytical operation. Focus upon the accuracy and precision of liquid handling and its laboratory-wide effect upon flexible operation and reproducibility for LBA methods that will be routinely employed over several years was one of the major reasons for choosing modular platforms over TLA. The breakdown of the preparation of standard calibrators/QCs/reagents and assay steps on to separate systems while avoiding the integration of periphery equipment, for example, plate washers, has provided maximal flexibility of accessibility for bench scientists via the path of shortened time of operation of the modular automated liquid handling system during a typical 8-h working day. Some of the systems were designed to have a focus upon the preparation of QCs, 
standard calibrators and the dilution of clinical and nonclinical study samples. Other modules were designed for the delivery of liquid to multiwell plates. The selection of deck space, in other words, types and numbers of labware accommodated by a given workstation, was reflective of the scalability of not only current and future sample processing requirements, but also to provide space to expand the daily experimental runs condensing the total length of method development time. The outcome of this setup was the successfully shorter development durations of bioanalytical methods. In direct comparison to meet this annual throughput, the flexibility of operation and efficiencies we needed would not be met by the equivalent cost of a single TLA with the required capacity. Modular automation systems have allowed us to switch between different methods easily with minimal interruption during normal working hours. Additionally, the number of samples can vary dramatically from a couple of hundreds (e.g., from nonclinical studies or cohort by cohort analysis from early phase of clinical studies) to thousands of samples from late-stage pivotal or Phase III studies. A TLA system is more suitable for routine and repeated testing with large numbers of samples, while modular systems will be much flexible and efficient to accommodate bioanalysis to meet the needs from different types of studies.

\section{Applications for laboratory automation \\ Reagent screening/pairing}

The quality of critical reagents, for example, specificity, selectivity and consistency between batches, is essential for the good performance of all LBA-based bioanalytical methods for supporting biologics [8-11]. The rapid generation and selection of these reagents such as anti-idiotypic ('anti-Id') antibodies often form the primary start point of the critical path of method development. Subsequent screening and pairing of hundreds of anti-Ids from hybridomas can be lengthy and labor-intensive. For example, PK method development generally requires optimal pairs of anti-Ids without significant matrix interference. Applying a standard 'checker board' design on 96-well plates using automated liquid handling, many anti-Id reagents can be screened and paired in days. In our laboratory, we typically run 8-point standard calibrator curves prepared in assay buffer without matrix and in diluted matrix to evaluate standard calibrator linearity and potential matrix interference with anti-Id pairs in a single experiment. One analyst can screen up to 72 pairs of conjugated anti-Ids for PK method use with standard 96-well plate format in a single day.

With multiplexing capabilities, such as U-plex plates from Meso Scale Discovery (MSD ${ }^{\circledR}$; MD, USA), the output of anti-Ids pairing screen can be improved to 432 pairs by one analyst on a single day. The data can be imported into a standard macro spreadsheet template for further analysis based on predefined assay selection criteria, such as standard curve linearity and slope, signal to noise ratio, etc. Automated liquid handling not only reduces the labor intensity of screening these reagents but provides more consistent data which can be compared across experiments for optimal selection of critical reagent pairs. Similar process can also be adopted into reagent selection for ADA and TE method development with slightly different outcomes and readouts.

\section{Automated liquid handling-assisted PK method development}

Drug concentration measurement is an essential component of pharmaceutical development since it directly impacts the establishment of correlation between toxicity/or efficacy and exposure during nonclinical and clinical studies. PK results are generated and compared across multiple studies over years of preclinical and clinical development. This demands high quality of PK results which in turn requires highly reproducible and robust PK methods. Several factors can directly impact the accuracy and precision of a PK method: preparation of standard calibrators and QC samples; QC and study sample dilutions; reagent preparation and additions. Duo et al. describe in detail in setting up a modular approach in using LBAs for discovery [12]. Modular automated liquid handling systems can also be adopted in these steps to improve the consistency of PK method performance in a clinical setting. Large volumes of standard calibrators ( $>5 \mathrm{ml}$ per calibrator) and QCs ( $>20 \mathrm{ml}$ per QC level) can be reproduced consistently on automation instrument to cover bioanalysis for large studies once storage and processing stability is demonstrated in method validation. With reproducible preparation of standard calibrators and QC samples, automation not only speeds up method development but also achieves a higher probability of success in subsequent method validations and 'laboratory to laboratory' method transfers if needed, with greater intralaboratory and interlaboratory ruggedness [5].

Automated liquid handling is also very useful in preparing diluted test samples of QCs and study samples. Different automation scripts need to be written and verified for different matrices, labware and liquid handling parameters ('liquid class'). It is very critical that each dilution scheme is verified for accuracy since any minor 
variation in each dilution scheme can be exponentially amplified during serial dilutions with same dilution scheme (e.g., performing consecutive 1:10 dilutions multiple times) or in combination with other dilution schemes. With improved precision of pipetting from automated liquid handling, consistency in mixing frequency/volumes lead to more reproducible assay performance. After sample dilution, the transfer of diluted samples and subsequent reagent addition are generally performed with a 96-well multichannel head due to its flexibility to work in 96- and 384-well plate formats. Groschl et al. demonstrate the use of certain software to simplify the process for aiding the analyst to produce curves on the Tecan Evo [13]. We have taken a similar process by using proprietary designed software where the user only enters three inputs: stock concentration, a reference concentration and curve volume. This has streamlined curve development by removing automation specialists. While worklists have been applied to automation successfully [12], we have found them cumbersome for analysts and thus have adapted this user-friendly software approach and extended it to QCs and sample dilution. Developers only need to enter stock concentrations for QC production and volumes for sample dilution. The software also performs all calculations for determining volumes for QC productions. With this software, we can have multiple analysts developing automated assays and are less dependent on automation specialists. Multiple publications have demonstrated the benefits of using 'design of experiments (DOE)' approach with factorial design to identify the optimal LBA conditions and reduce assay variations [14]. However multifactor DOE experimental runs can be very complex, difficult and prone to error when performed manually, which further complicates the readout and decision-making. With an automated liquid handler, these DOE run templates can be easily adapted to an automation instrument which in turn can improve the consistency and outcome of DOE experimental runs [1]. Wang et al. successfully used DOE to identify three specific assay steps that would greatly benefit from the use of automation and applied modular automation to those steps [15]. A common approach to clinical automation is to take a developed or validated assay and to automate it or certain process of it de facto [15]. We have taken the novel approach of applying automation at all aspects of development. Multiparameter experiments are large scale (up to 12 plates) single experiments executed with automated liquid handling each focusing upon key assay variables, such as reagent concentrations, buffer components, minimal required dilutions, various levels of QCs and ranges of standard calibrators. Standard calibrators covering different dynamic ranges and their corresponding QC samples at different levels can be prepared with automated liquid handlers. This multiparameter assessment with an automated liquid handler can significantly reduce the method development time and improve the consistency in assay results, which results in faster decision-making and trouble shooting. Over tens of thousands of data points are generated from this process which is then imported into a predefined macro spreadsheet for analysis. The selection of critical reagent concentrations, buffer components and minimal required dilutions can be greatly optimized in the context of the performance in accuracy and precision from standard calibrators and QC samples. Since these assay parameters are screened, selected and optimized on automated liquid handlers, the probability of scalability of these LBA methods with automation is then greatly increased.

\section{Automated liquid handling-assisted ADA method development}

LBA methods for ADA evaluation are semiquantitative and will use the signal generated (this value can be normalized to an internal control) from the assay to directly determine the potential immunogenicity status of a sample. One of the unique $\mathrm{ADA}$ assay challenges is drug tolerance. Current regulatory requirement defines an $\mathrm{ADA}$ assay sensitivity at $100 \mathrm{ng} / \mathrm{ml}$ while most of therapeutic drugs will have much higher $C_{\max }$ and trough concentrations, usually at $\mu \mathrm{g} / \mathrm{ml}$ or higher. Standard ADA screening methods utilize drug itself as assay capture and detection reagents to detect ADAs in study samples which will experience assay interference from circulating drug present in study samples [16,17]. Similarly, target proteins which the drug binds can also cause interference in ADA methods [18].

One of the standard approaches to eliminate drug/or target interference in ADA methods is acid dissociation [19]. Study samples are treated with a low $\mathrm{pH}$ buffer to dissociate ADA-drug complexes. Sufficient dissociation of drugADA complexes in serum samples will depend on different acid types, molar concentration of acid, $\mathrm{pH}$, volume ratio between samples and acid buffer and duration of treatment [19]. To determine the optimal conditions manually is laborious and subject to assay variability. Akin to the way in which we have applied automated liquid handling to our PK assay development, the same was performed for our ADA assays. The ability to test numerous buffers and acid conditions simultaneously has required a larger scale single experiment and therefore a 384-well format has greatly facilitated this process, turning weeks of experiments into days with improved data output and reduced development time. Selection of optimal acid dissociation conditions can be performed with the assistance of automated liquid handling with a templated design. The consistency of pipetting from automated liquid handlers 
makes the runs operationally achievable and reduces manual errors which can obscure experimental data resulting in suboptimal selections. Once the optimal acid dissociation conditions are selected, the rest of ADA method development can be adopted on to automated liquid handlers with sample dilutions and reagent addition. Data analysis can be performed with an automated macro spreadsheet with improved efficiency.

\section{Considerations in method validation \& bioanalysis}

All bioanalytical methods should be developed, validated and implemented according to regulatory requirements and industry standards $[1-4,20-29]$ for preclinical and clinical studies assessing safety and efficacy. There are other considerations when utilizing automated liquid handling in regulated bioanalysis. First, all the scripts from automated liquid handler used in each method, such as preparation of standard calibrators/QCs, dilution/transfer of samples, reagent additions, etc., should have different secure levels of user privileges according to 21CFR part 11 regulations. The automated scripts should be documented in the validation report of the methods and listed in the relevant standard operating procedures of the methods. The application of automated liquid handling either as workstation or TLA approach does facilitate the opportunity to perform bioanalysis at a greater scale than is routinely run with manual pipetting. There are two approaches to bioanalysis that are somewhat dependent upon the configuration of workstation equipment, batch or linear processing of assay plates. Batch processing is where the step is completed for all plates and the liquid contact time can vary due to controlling incubation times per set of plates, while linear processing is a process of individually controlling the contact and incubation time through controlling incubation times per plate.

In our laboratory application of the modular workstation approach of automation, the batch format is the preferred process. Batch size testing is not a requirement in the regulatory guidance, but to understand the scalability and performance limits of LBA methods is critical for improvement in sample throughput and to monitor plate passing rate during bioanalysis, when these LBA methods are being implemented. The scalability of LBA methods with automation depends on the relationship between processing or contact time of liquid in plate and stability of immunocomplexes (analyte and its specific reagents) formed on assay plates. In our laboratory, we have adopted two approaches to increase the number of assay plates during batch size evaluation. One approach is to test and stress the assay plates to the maximal designated deck size a given automated liquid handler can accommodate in a single run of the method up to the deck plate capacity, till the failure of a given assay plate. This is the most straightforward approach but it requires very high stability of the assay since the time from the first plate processed to the last plate completed can be very different, as long as $1 \mathrm{~h}$ on our systems. This larger single-run batch size will free up hands-on time, but will limit the flexibility of accommodating many bench scientists and their numerous methods with a limited number of workstations. A second strategy of a 'rolling batch' processing is often the better alternative. In this approach, a large number of plates (such as thirty-six 96-well assay plates) can be divided into several sub-batches with a small number of plates, for example, six plates per sub-batch. As soon as the first six-plates are off-deck, the second six-plates can be initiated and so on till all six sub-batches are completed. This approach requires good stability of assay reagents and study samples on deck during processing, for example, stability at room temperature up to $24 \mathrm{~h}$, then theoretically as many as six-plate sub-batch runs can be processed in a rolling fashion within $24 \mathrm{~h}$. This is another reason in our laboratory we prefer modular automation system since it provides the maximal flexibility for different scalability. Automated liquid handling systems significantly increase the throughput of LBA methods by increased runs of large number of plates. This can also be achieved manually, but not without errors and the added physical and mental stresses to the bench scientist.

\section{Future perspective}

There is greater business demand on bioanalytical laboratories to be more flexible and high throughput to deliver bioanalytical data with high quality and speed for quick decision-making during drug development. This process in turn demands LBA methods to be highly accurate, precise and robust. Current immunoassay platforms still have certain limitations which impede scalability, while script writing for automation instrument generally requires special programming skills, which often are on the critical path when adopting automation during method development. The emergence of a variety of novel immunoassay platforms continuously improve the robustness and sensitivity of LBA methods, which can provide both opportunities and challenges when interfacing with robotic platforms. Automation instrumentation is evolving with more user-friendly interfaces and robotics that have improved functionality and speed. The application of TLAs controlled through artificial intelligence will result in shortened assay development times, larger scale bioanalysis and faster drug development. Ultimately, automation 
of LBA methods is the path and the future of bioanalytical laboratories to meet the needs of drug development. In the time being, the modular workstation approach to automation leads to the most flexibility while providing more robust assays, faster development times, and increased throughput of bioanalysis.

\section{Financial \& competing interests disclosure}

The authors have no relevant affiliations or financial involvement with any organization or entity with a financial interest in or financial conflict with the subject matter or materials discussed in the manuscript. This includes employment, consultancies, honoraria, stock ownership or options, expert testimony, grants or patents received or pending, or royalties.

No writing assistance was utilized in the production of this manuscript.

\section{References}

Papers of special note have been highlighted as: $\bullet$ of interest; $\bullet \bullet$ of considerable interest

1. Joelsson D, Moravec P, Troutman M, Pigeon J, Dephillips P. Optimizing ELISAs for precision and robustness using laboratory automation and statistical design of experiments. J. Immunol. Methods 337(1), 35-41 (2008).

2. Ahene A, Calonder C, Davis S et al. Automation practices in large molecule bioanalysis: recommendations from group L5 of the global bioanalytical consortium. AAPS J. 16(1), 164-171 (2014).

3. Allinson JL, Blick KE, Cohen L, Higton D, Li M. Ask the experts: automation: part I. Bioanalysis 5(16), 1953-1962 (2013).

4. Armbruster DA, Overcash DR, Reyes J. Clinical chemistry laboratory automation in the 21 st century - amat victoria curam (victory loves careful preparation). Clin. Biochem. Rev. 35(3), 143-153 (2014).

5. Bock JL. The new era of automated immunoassay. Am. J. Clin. Pathol. 113(5), 628-646 (2000).

6. Li M. Automation in the bioanalytical laboratory: what is the future? Bioanalysis 5(23), 2859-2861 (2013).

- Guidelines for robotic systems in a regulated bioalanalytical setting.

7. Palandra J, Weller D, Hudson G et al. Flexible automated approach for quantitative liquid handling of complex biological samples. Anal. Chem. 79(21), 8010-8015 (2007).

8. Geist BJ, Egan AC, Yang TY, Dong Y, Shankar G. Characterization of critical reagents in ligand-binding assays: enabling robust bioanalytical methods and lifecycle management. Bioanalysis 5(2), 227-244 (2013).

9. Haulenbeek J, Piccoli SP. Conjugated critical reagent characterization for ligand-binding assays: using MALDI-TOF-MS as an orthogonal tool to assess assay performance. Bioanalysis 6(7), 983-992 (2014).

10. O’hara DM, Theobald V. Life cycle management of critical ligand-binding reagents. Bioanalysis 5(21), 2679-2696 (2013).

11. O'hara DM, Theobald V, Egan AC et al. Ligand binding assays in the 21 st century laboratory: recommendations for characterization and supply of critical reagents. AAPS J. 14(2), 316-328 (2012).

12. Duo J, Dong H, Desilva B, Zhang YJ. A generic template for automated bioanalytical ligand-binding assays using modular robotic scripts in support of discovery biotherapeutic programs. Bioanalysis 5(14), 1735-1750 (2013).

13. Groschl M, Markus A, Simone L et al. A liquid handling robot for robust and reproducible preparation of standard and quality control samples in bioanalysis. Adv. Robot. Automat. 6(1), 160 (2017).

14. Ray CA, Patel V, Shih J et al. Application of multi-factorial design of experiments to successfully optimize immunoassays for robust measurements of therapeutic proteins. J. Pharm. Biomed. Anal. 49(2), 311-318 (2009).

15. Wang J, Patel V, Burns D et al. Laboratory automation of high-quality and efficient ligand-binding assays for biotherapeutic drug development. Bioanalysis 5(13), 1635-1648 (2013).

16. Gunn GR, 3rd, Sealey DC, Jamali F, Meibohm B, Ghosh S, Shankar G. From the bench to clinical practice: understanding the challenges and uncertainties in immunogenicity testing for biopharmaceuticals. Clin. Exp. Immunol. 184(2), 137-146 (2016).

- Detailed use of modular automated systems in a laboratory.

17. Zoghbi J, Xu Y, Grabert R, Theobald V, Richards S. A breakthrough novel method to resolve the drug and target interference problem in immunogenicity assays. J. Immunol. Methods 426, 62-69 (2015).

18. Dai S, Schantz A, Clements-Egan A, Cannon M, Shankar G. Development of a method that eliminates false-positive results due to nerve growth factor interference in the assessment of fulranumab immunogenicity. AAPS J. 16(3), 464-477 (2014).

19. Kavita U, Duo J, Crawford SM et al. A systematic study of the effect of low $\mathrm{pH}$ acid treatment on anti-drug antibodies specific for a domain antibody therapeutic: Impact on drug tolerance, assay sensitivity and post-validation method assessment of ADA in clinical serum samples. J. Immunol. Methods 448, 91-104 (2017).

- Comprehensive look at how automation can be used and improve assay performance in a regulated laboratory.

20. EMA. Guideline on Bioanalytical Method Validation (2012). www.ema.europa.eu/docs/en_GB/document_library/Scientific_guideline/2011/08/WC500109686.pdf 
21. US FDA. Draft Guidance for Industry: Bioanalytical Method Validation (2013). www.fda.gov/downloads/drugs/guidances/ucm368107.pdf

22. Desilva B, Smith W, Weiner R et al. Recommendations for the bioanalytical method validation of ligand-binding assays to support pharmacokinetic assessments of macromolecules. Pharm. Res. 20(11), 1885-1900 (2003).

23. Imazato-Hirano $M$, Taniguchi $Y$, Kakehi $M$ et al. Japanese bioanalytical method validation guideline: the world's first regulatory guideline dedicated to ligand-binding assays. Bioanalysis 7(9), 1151-1156 (2015).

24. Mire-Sluis AR, Barrett YC, Devanarayan V et al. Recommendations for the design and optimization of immunoassays used in the detection of host antibodies against biotechnology products. J. Immunol. Methods 289(1-2), 1-16 (2004).

25. Shankar G, Devanarayan V, Amaravadi L et al. Recommendations for the validation of immunoassays used for detection of host antibodies against biotechnology products. J. Pharm. Biomed. Anal. 48(5), 1267-1281 (2008).

26. Wadhwa M, Bird C, Dilger P, Gaines-Das R, Thorpe R. Strategies for detection, measurement and characterization of unwanted antibodies induced by therapeutic biologicals. J. Immunol. Methods 278(1-2), 1-17 (2003).

27. European Medicine Agency. Guideline on Immunogenicity Assessment of Biotechnology-Derived Therapeutic Proteins (2006). www.ema.europa.eu/docs/en_GB/document_library/Scientific_guideline/2009/09/WC500003946.pdf

28. US FDA. Draft Guidance for Industry: Assay Development and Validation for Immunogenicity Testing of Therapeutic Protein Products (2016). www.fda.gov/downloads/Drugs/Guidances/UCM192750.pdf

29. US Pharmacopeia. General Chapter $<1106>$ Immunogenicity Assays - Design and Validation of Immunoassays to Detect Anti-Drug Antibodies (2013). https://www.usp.org/sites/default/files/usp/document/our-work/biologics/USPNF810G-GC-1132-2017-01.pdf 